\title{
Software Defined Radio-based Signal Detection and RF Parameter Estima- tion Platform for Enhancing Electrical and Computer Engineering Curricula
}

\section{Dr. Zhiping Zhang, Wright State University}

Zhiping Zhang received his B.S. degree in electrical engineering from Nankai University, Tianjin, China, in 2001, M.S. and Ph.D. degrees in intelligence science from Peking University, Beijing, China, in 2004 and 2011 respectively. From 2011 to 2013, he was a Postdoctoral Research Fellow at the Department of Computer Science and Technology, Peking University. Since 2013, he has served as a research faculty member and co-director of Broadband, Mobile and Wireless Networking Laboratory at the Department of Electrical Engineering of Wright State University.

\section{Dr. Zhiqiang Wu, Wright State University}

Dr. Zhiqiang Wu received his BS from Beijing University of Posts and Telecommunications in 1993, MS from Peking University in 1996, and PhD from Colorado State University in 2002, all in electrical engineering. He has worked at West Virginia University Institute of Technology as assistant professor from 2003 to 2005. He joined Wright State University in 2005 and currently serves as full professor. Dr. $\mathrm{Wu}$ is the author of national CDMA network management standard of China. He also co-authored one of the first books on multi-carrier transmission for wireless communication. He has published more than 100 papers in journals and conferences. He has served as Chair of Acoustic Communication Interest Group of IEEE Technical Committee on Multimedia Communications. His research has been supported by the National Science Foundation, Air Force Office of Scientific Research, Air Force Research Laboratory, Office of Naval Research, and NASA. His work on software defined radio implementation of cognitive radio won the Best Demo Award at IEEE Globecom 2010.

\section{Prof. Bin Wang, Wright State University}

Prof. Bin Wang earned his Ph.D. from the Ohio State University in 2000. He joined the Wright State University in September 2000, where he is currently full professor of computer science and engineering. His research interests include optical networks, real-time computing, mobile and wireless networks, cognitive radio networks, trust and information security, and semantic web. He is a recipient of the US Department of Energy Career Award. His research has been supported by US Department of Energy, National Science Foundation, Air Force Office of Scientific Research, Air Force Research Laboratories, Ohio Supercomputer Center, and the State of Ohio. 


\title{
Software Defined Radio based Signal Detection and RF Parameter Estimation Platform for Enhancing Electrical and Computer Engineering Curricula
}

\begin{abstract}
Supported by an NSF TUES type II grant, we have developed a software defined radio (SDR) based signal detection and radio frequency (RF) parameter estimation platform for enhancing electrical and computer engineering curricula. Specifically, we have developed an SDR based signal detection and RF parameter estimation platform which can be adopted by many courses of electrical and computer engineering curricula. This SDR based platform is equipped with a user-friendly graphical user interface (GUI). Students can easily adjust the center frequency and bandwidth of the RF spectrum and choose different signal detection/RF parameter estimation algorithms. We have integrated a suite of signal detection and RF parameter estimation algorithms in the platform, including energy based detection, cyclostationary analysis based detection, fourth order cumulants based detection, etc. The platform is capable of detecting unknown RF signals, estimating their RF parameters such as carrier frequency, symbol rate, and modulation type. This platform can be adopted by many undergraduate and graduate level courses of electrical and computer engineering. The userfriendly GUI of this platform makes it a useful tool to attract high school students and freshmen year students into the STEM fields. The light weight and portable nature of the SDRs allow this platform to be easily transferable from one place to another, making it an attractive tool for capstone senior design teams as well. Through collaboration among the three participating institutions (including a Historically Black College), the developed SDR based signal detection and RF parameter estimation platform will be integrated in undergraduate curricula of all three institutions.
\end{abstract}




\section{Introduction}

We are living in a world of wireless communication and networking. More than $90 \%$ of the US population use wireless services such as cell phones and WiFi every day ${ }^{[1][2]}$. Consequently, communication and networking has increasingly become an important part of modern electrical engineering and computer science/engineering curricula ${ }^{[3]}$. In our previous National Science Foundation (NSF) funded Course, Curriculum, and Laboratory Improvement (CCLI) project "Evolvable wireless laboratory design and implementation for enhancing undergraduate wireless engineering education", we have developed and demonstrated the first nationwide example of evolvable software defined radio (SDR) based laboratories for three existing undergraduate courses ${ }^{[4][5]}$. Given the success of this project, NSF has continued to support us through a Transforming Undergraduate Education in Science (TUES) type II project to further develop a series of SDR based lab modules that serve a wide range of courses from a freshman year introductory course to senior year capstone design projects.

In this paper, we report an SDR ${ }^{[6]-[11]}$ based signal detection and RF parameter estimation platform which can be adopted by many courses of electrical and computer engineering curricula. This SDR based platform is equipped with a user-friendly graphical user interface (GUI). Students can easily adjust the center frequency and bandwidth of the RF spectrum and choose different signal detection/RF parameter estimation algorithms. The GUI also displays the RF signal captured by the SDR, the instantaneous spectrum, statistical analysis of the signal such as cumulative distribution function (CDF). We have integrated a suite of signal detection and RF parameter estimation algorithms in the platform, including energy based detection, cyclostationary analysis based detection, fourth order cumulants based detection, etc. The platform is capable of detecting unknown RF signals, estimating their RF parameters such as carrier frequency, symbol rate, and modulation type.

This platform can be adopted by many undergraduate and graduate level courses of electrical and computer engineering. Such courses include digital communication and wireless communication, microwave technology, radar, antenna design, communication network, cyber security, etc. The user-friendly GUI of this platform makes it a useful tool to attract high school students and freshmen year students into the STEM fields. The light weight and portable nature of the SDRs allow this platform to be easily transferable from one place to another, making it an attractive tool for capstone senior design teams as well.

Through collaboration among the three participating institutions (Wright State University, Miami University (a mostly undergraduate serving institution), and Central State University (an 
HBCU)), the developed SDR based general modulation/demodulation platform will be integrated in undergraduate curricula of all three institutions.

\section{Software Defined Radio Platform}

Figure 1 illustrates the block diagram of the transmitter and receiver of software defined radio. As shown in the figure, in software defined radio, most of the signal processing is done directly in the software domain instead of the hardware domain. At the SDR transmitter, user can easily adjust the communication waveform by modifying the software, and a digital to analog convertor will generate the resulting analog waveform. Similarly, at the SDR receiver, the demodulation and decoding of the received communication signal is performed in software after analog to digital conversion. Therefore, the SDR platform offers higher flexibility and evolvability than their hardware counterparts.

Receive Path:

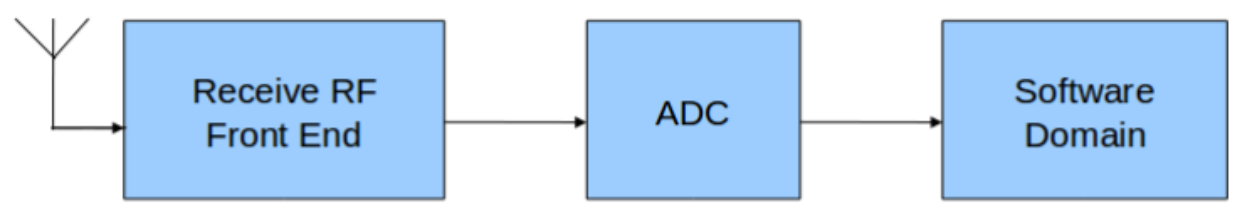

Transmit Path:

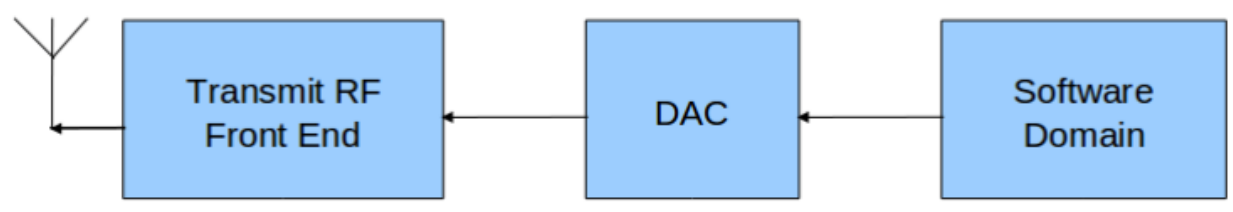

Figure 1. Software Defined Radio

We have chosen the universal software radio peripheral (USRP) ${ }^{[10][11]}$ hardware by ETTUS and National Instruments because of its popularity in academia and its affordability. We have implemented the signal detection and RF parameter estimation platform on four different USRP motherboards: USRP1, USRP2, USRP N210, and USRP X300. We have chosen the WBX daughterboard because of its wide bandwidth (from $50 \mathrm{MHz}$ to $2.2 \mathrm{GHz}$ ) and its dual transmitter/receiver functionality. We have also used a complete wireless test and measurement system including vector signal generator, arbitrary waveform generator, vector spectrum analyzer, wireless channel emulator and digital phosphor oscilloscope to validate and expedite the development of the SDR based signal detection and RF parameter estimation platform. Figure 2 shows the four USRP boards and the wireless test and measurement system. It is important to note that the developed SDR based signal detection and RF parameter estimation 
platform and associated laboratories do not depend on the wireless test and measurement system. The wireless test and measurement system was used to help and expedite the development of our laboratories only. The SDR based platform and laboratories only require low cost software defined radio boards to fully function.

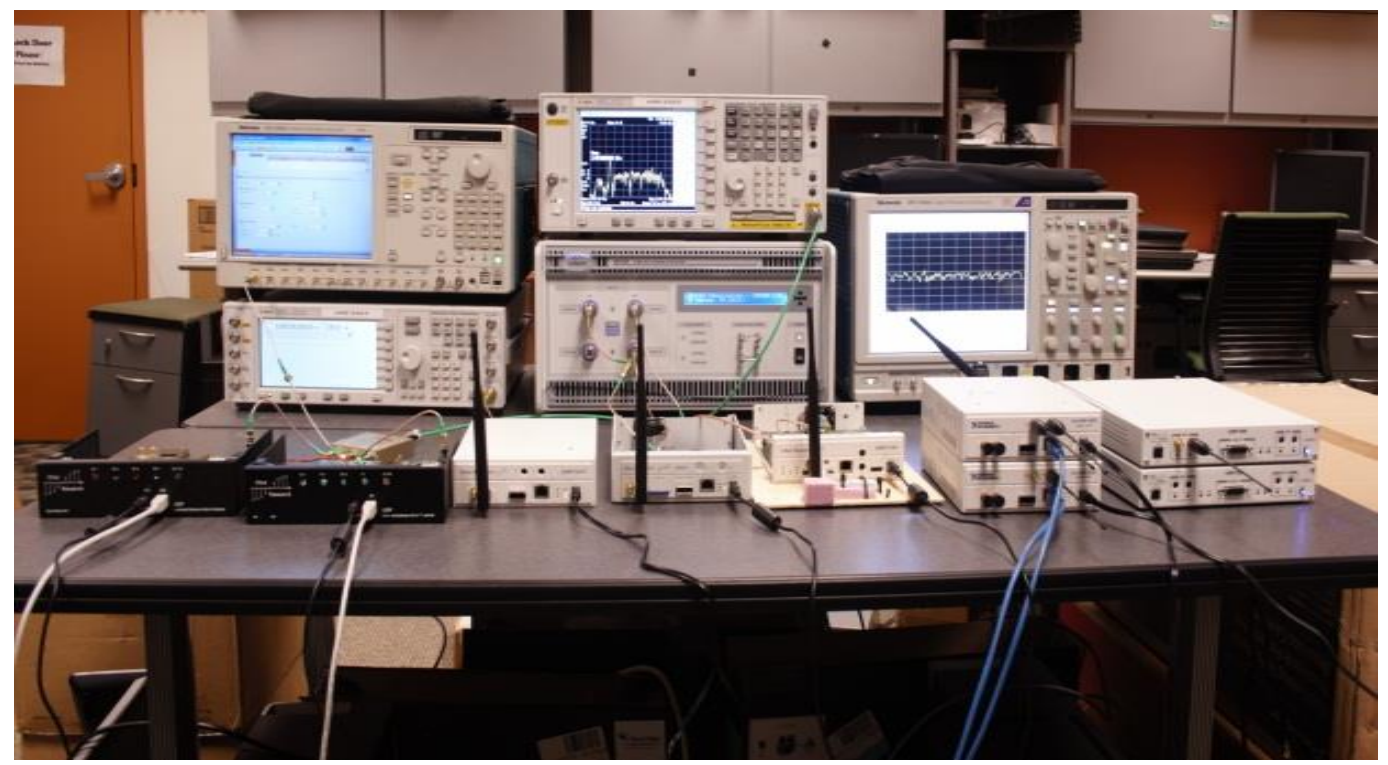

Figure 2. SDR Boards and Wireless T\&M System

\section{SDR based Signal Detection and RF Parameter Estimation Platform}

Signal detection and estimation is a very important course in electrical and computer engineering curriculum. However, most of existing courses on signal detection and estimation are taught without a lab: only theoretical analysis and numerical simulation are used. We have developed a SDR based signal detection and RF parameter estimation platform to solve this problem. The developed platform can be adopted by the signal detection and estimation course and other communication/networking/signal processing courses to give students intuitive guidance and hands-on experiences.

We have used a combination of GNU radio, Python, and Matlab to develop a user friendly signal detection/RF parameter estimation platform on SDR. This SDR based platform is equipped with a user-friendly graphical user interface (GUI). Students can easily adjust the center frequency and bandwidth of the RF spectrum and choose different signal detection/RF parameter estimation algorithms. Figure 3 shows the GUI of the platform. As shown, students can easily adjust important RF parameters such as center frequency, observation bandwidth, sampling rate, etc. We have also developed an embedded channel emulator through which students can simulate additive white Gaussian noise (AWGN) channel, Rayleigh flat fading channel, and multi-path 
fading channel. Students can also adjust the noise level to simulate different signal to noise ratios (SNRs).

A suite of signal detection and RF parameter estimation algorithms have been developed and integrated in the SDR platform. As an example, students can specify a band of interest and perform spectrum sensing and signal detection first, then estimate the important RF parameters such as carrier frequency, symbol rate, and modulation schemes on the detected signals.

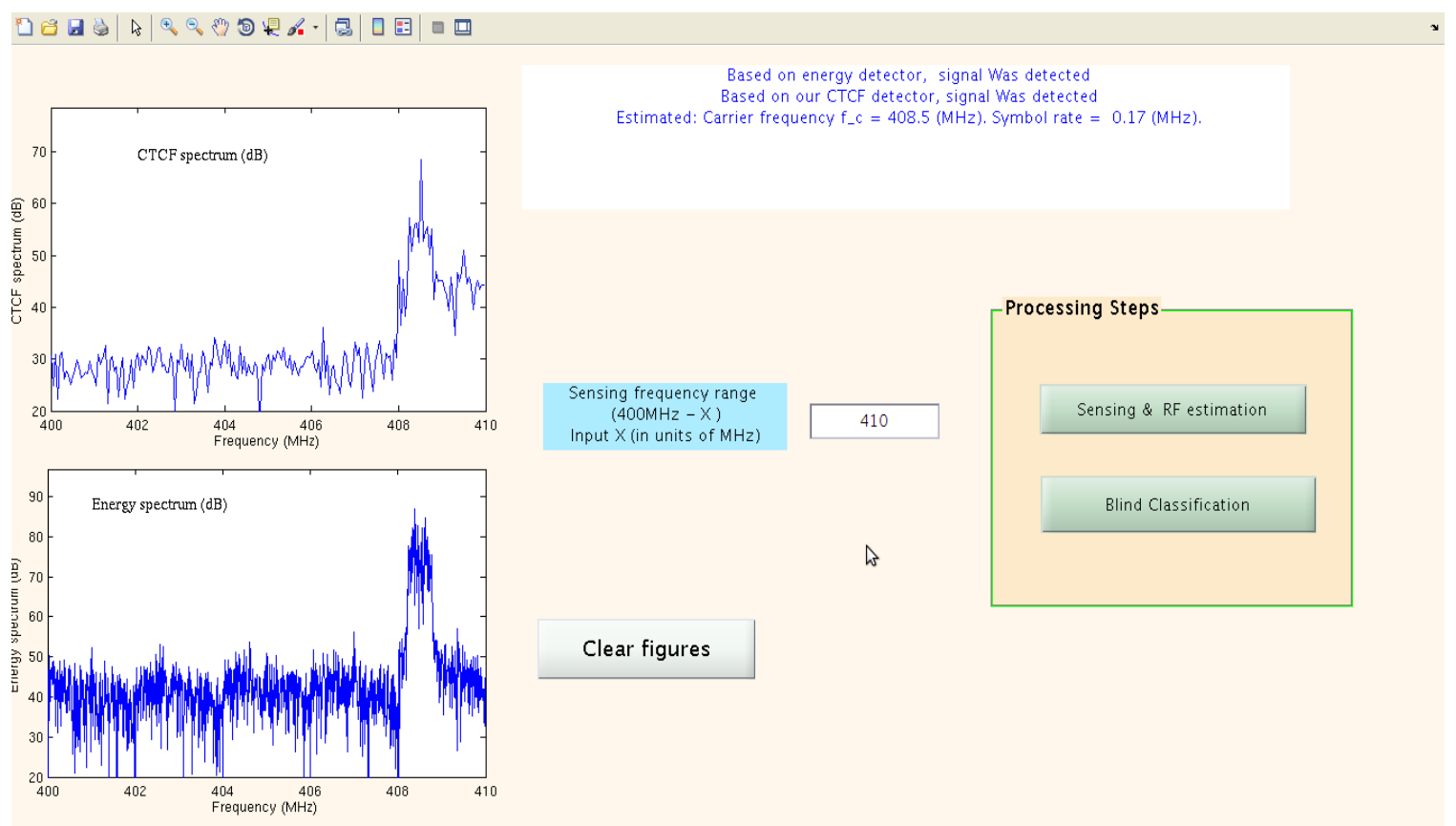

Figure 3. GUI of Signal Detection/RF Estimation Platform

Figure 4 shows a snapshot of a spectrum sensing/signal detection result on the SDR platform over the TV band centered at $448 \mathrm{MHz}$. As shown in Figure 4, three narrowband signals (represented by three different colors) are detected in the band of interest. In the example shown in Figure 4, an energy based detection algorithm is employed and a pre-determined threshold is used to distinguish signal from noise. 


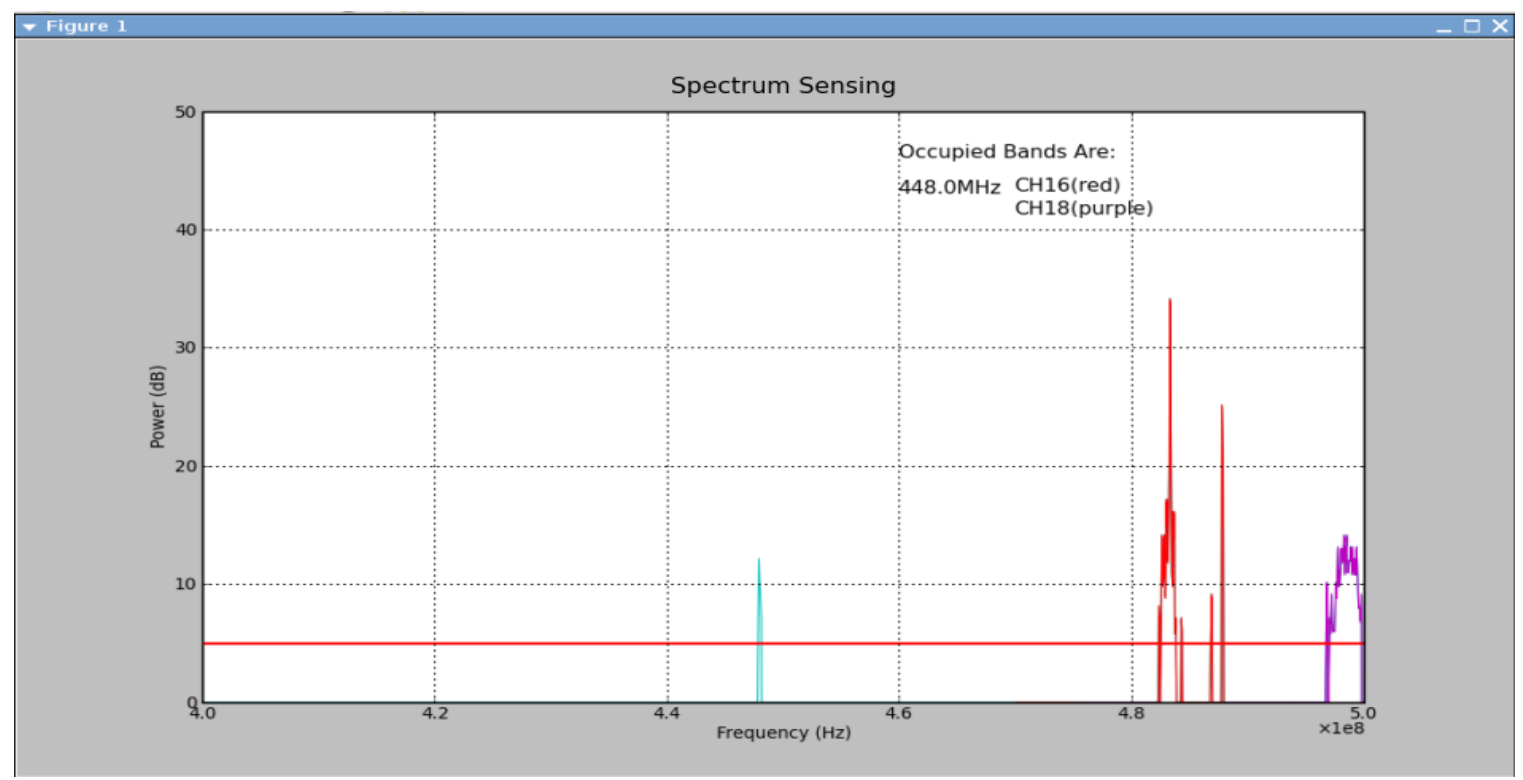

Figure 4. Signal Detection

After the signal detection stage, the SDR based platform performs RF parameter estimation. Figure 5 illustrates an example of carrier frequency and symbol rate estimation through cyclostationary analysis. Specifically, a cyclic temporal cumulant function (CTCF) based algorithm is computed in real time to estimate the carrier frequency and symbol rate of the detected narrowband signal. The estimated results are displayed on the GUI.

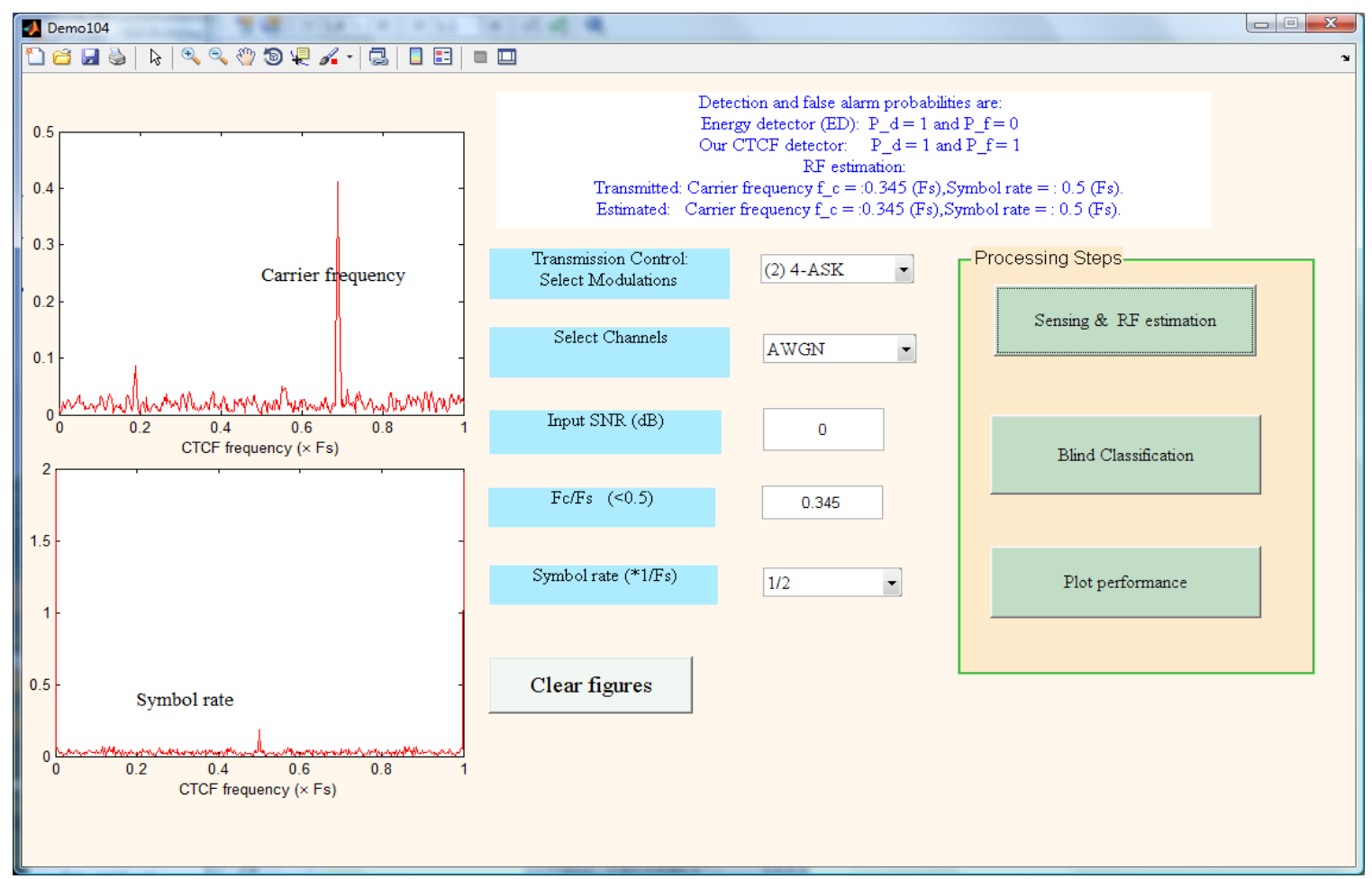

Figure 5. RF Parameter Estimation 
Next, the modulation scheme of the detected signal is also estimated and the reconstructed constellation is displayed on the GUI. Figure 6 shows such an example of a reconstructed QPSK modulation constellation. The top constellation was reconstructed using perfect channel information, and the bottom one was reconstructed through blind channel estimation and equalization.

\section{Enhancing Curricula}

The developed SDR based signal detection/RF parameter estimation platform has become a very useful tool to enhance the communication and networking curricula at the three participating institutions of this NSF TUES type II project. The platform is very user friendly through its GUI and its graphical display of the signal detection/spectrum sensing/RF estimation/modulation classification results. During the 2014-2015 academic year, labs built upon this platform have been adopted at Wright State University in multiple courses such as EE4210/6210 Digital Communication, EE4730/6730 Wireless Communication, EE7350 Wireless Communication Techniques, EE7360 Advanced Wireless Communication Techniques, and EE8000 Advanced Electronic Warfare. We will extend these labs to the other two participating institutions of our project during the 2015-2016 academic year.

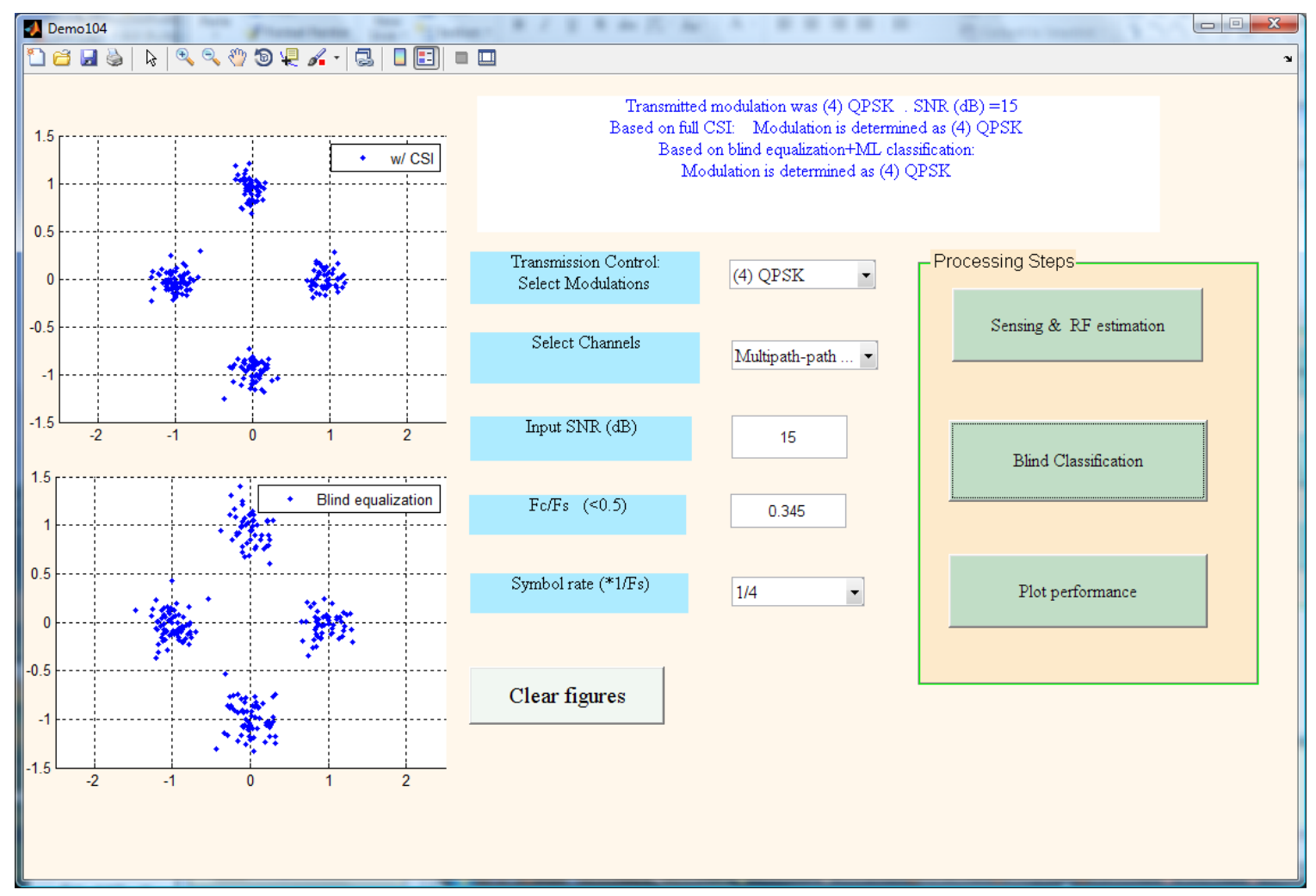

Figure 6. Modulation Constellation Reconstruction 
The platform provides students with an intuitive understanding of sophisticated signal detection and estimation algorithms and underlying mathematical theories. By adjusting RF parameters, varying SNRs and changing channels, students are excited and gain important hands-on experiences. During the course review, we have received universal positive feedback on the labs and the SDR based platform.

Specifically, during the EE4210 Digital Communication and associated EE4210L lab courses in Fall Semester of 2015, we have conducted an anonymous survey to all students about the effectiveness of the developed SDR based platform. The survey was handed to students during class of the last week and students were advised to fill in the survey and hand it in without presence of the instructor. Question 1 of the survey asked students to provide a numerical score on a scale of 1 to 5 on the effectiveness of the SDR based teaching lab platform to improve student's understanding of communication theory. Out of 69 students of 7 lab sections, 50 of them returned the survey. The following table summarizes the score given by students:

\begin{tabular}{|l|l|l|c|c|c|}
\hline & 1 & 2 & 3 & 4 & 5 \\
\hline \# of students & 2 & & 14 & 14 & 20 \\
\hline
\end{tabular}

Table I. Student Evaluation Score

As shown in Table I, the majority of the students who responded to the survey have given a high score to the effectiveness of the developed SDR platform. The mean of the score is 4 , the standard deviation is 1.04 .

Due to the graphical manner of the signal detection and modulation classification, this platform is also a useful tool to attract high school students and first year undergraduate students into the STEM fields. We have shown this platform to multiple groups of visiting high school students and incoming freshman year students, and received strong interest from them to join different engineering majors. It is interesting and important to note that although the high school students and freshman year students do not have the mathematical and technical background to understand all details of the platform, the graphical manner of the platform and the interactive GUI enable them to enjoy the appreciate the power of communication and signal processing.

\section{Conclusions}

Supported by an NSF TUES type II grant, we have developed a software defined radio (SDR) based signal detection and RF parameter estimation platform for enhancing undergraduate and 
graduate communication and networking curricula. The SDR based platform allows students to observe the signal detection and estimation theory in an intuitive manner. Students can easily adjust RF parameters, SNRs, and channel models through a GUI. We have developed a series of laboratories on this platform and integrated them in multiple courses across three participating institutions.

\section{References:}

[1] U.S. Wireless Quick Facts, CTIA-The Wireless Association, http://www.ctia.org/advocacy/research/index.cfm/aid/10323

[2] IEEE 802.11 standard, http://standards.ieee.org/about/get/802/802.11.html

[3] Option in Wireless Engineering, College of Engineering and Computer Sciences, Wright State University, http://www.cs.wright.edu/cecs/academic-programs.shtml

[4] B. Wang, Y. Pei and Z. Wu, "Software Radio Based Wireless Laboratory Design and Implementation for Enhancing Undergraduate Wireless Engineering Education," Proceedings of 2010 Annual ASEE Conference and Exposition, Louisville, KY June 2010.

[5] B. Guenther, B. Rahn, B. Wang and Z. Wu, "Real-Time Video Transmission from High Altitude Balloon: An Interdisciplinary Senior Design Project," Proceedings of 2010 Annual ASEE Conference and Exposition, Louisville, KY June 2010.

[6] Software Defined Radio Forum, http://www.sdrforum.org

[7] M. Dillinger, K. Madani and N. Alonistioti, Software Defined Radio: Architectures, Systems and Functions, Wiley, 2003

[8] W. Tuttlebee, Software Defined Radio: Enabling Technologies, Wiley, 2002

[9] P. Kenington, RF and Baseband Techniques for Software Defined Radio, Artech House Publishers, 2005

[10] Universal Software Radio Peripheral, http://www.ettus.com/

[11] GNU Radio, http://gnuradio.org 